\title{
A Molar Pregnancy Detected by Following $\beta$-Human Chorionic Gonadotropin Levels after a First Trimester Loss
}

\author{
Lee T. Dresang, MD
}

This case report summarizes the sequence of events that led to the detection of a molar pregnancy missed by ultrasound and initial pathology examinations. This patient illustrates that following serial quantitative $\beta$-human chorionic gonadotropin (B-hCG) levels after spontaneous, therapeutic, and elective abortions will help detect potentially life-threatening molar and ectopic pregnancies. Further research is needed to determine the optimal frequency of B-hCG testing. For now, maternity care providers can follow the American College of Obstetrics and Gynecology recommendation that abnormal bleeding for more than 6 weeks after any pregnancy should be evaluated with B-hCG testing. Earlier testing may be considered. (J Am Board Fam Pract 2005;18:570-3.)

In December 2001, a patient with first trimester bleeding had an irregular intrauterine gestational sac containing an 8-week-sized fetus with no heart rate on ultrasound (Figure 1). The patient opted for a dilatation and curettage $(\mathrm{D} \& \mathrm{C})$ over expectant management. Her $\beta$-human chorionic gonadotropin (B-hCG) was 43,005 before the procedure. Ten days after the procedure, it was 1,285 , and the pathologic diagnosis was "products of conception." When the patient returned a week later, her BhCG had increased unexpectedly to 2,572 .

The differential diagnosis included a new pregnancy, retained products of conception, a heterotopic pregnancy and a molar pregnancy. A transvaginal ultrasound showing an empty uterus, normal adnexa, and no fluid in the cul-de-sac ruled out a new normal pregnancy. A gestational sac should be visible on transvaginal ultrasound with a B-hCG above 2,000. A consultant recommended a repeat D\&C. The B-hCG was 2991 week after the repeat procedure, and 2 weeks later, it was negative. However, pathology could not rule out a molar pregnancy; both D\&C specimens were sent to the Mayo Clinic, and a diagnosis of a partial hydatidi-

Submitted, revised, 30 March 2005.

From the University of Wisconsin-Madison Medical School, Department of Family Medicine, St. Luke's Family Practice Residency, Milwaukee, WI 53207

Conflict of interest: none declared.

Corresponding author: Lee T. Dresang, MD, University of Wisconsin Medical School, Department of Family Medicine, St. Luke's Family Practice Residency, 1230 W. Grant Street, Milwaukee, WI 53215 (e-mail: ldresang@fammed. wisc.edu). form mole was made. As advised, the patient had B-hCGs every week for 3 weeks and then every month for a year. All were negative, as was her chest radiograph. The patient used birth control for a year, as recommended.

In September 2003, the same patient presented with a new pregnancy and bleeding. Ultrasound revealed an irregular empty uterine sac and a quantitative B-hCG of 6888. She miscarried 2 days later. One week later, her B-hCG was 358 and negative since. Pathology and triploid placental tissue were consistent with another partial hydatidiform mole.

\section{Literature Review}

A Medline search was performed using the keywords "heterotopic pregnancy," and MeSH headings including: hydatidiform mole, chorionic gonadotropin, $\beta$ subunit, human, trophoblastic neoplasms, choriocarcinoma, and gestational trophoblastic neoplasms. In addition, the Cochrane database and American College of Obstetrics and Gynecology (ACOG) technical bulletins were reviewed.

\section{Discussion}

Both molar and heterotopic pregnancies can be fatal. Approximately $20 \%$ of complete moles and 0 to $3.5 \%$ of partial moles lead to persistent gestational trophoblastic disease (GTD). ${ }^{1}$ Although most patients with GTD can be successfully treated with chemotherapy, "there are still those who will die from this disease or receive inadequate treat- 


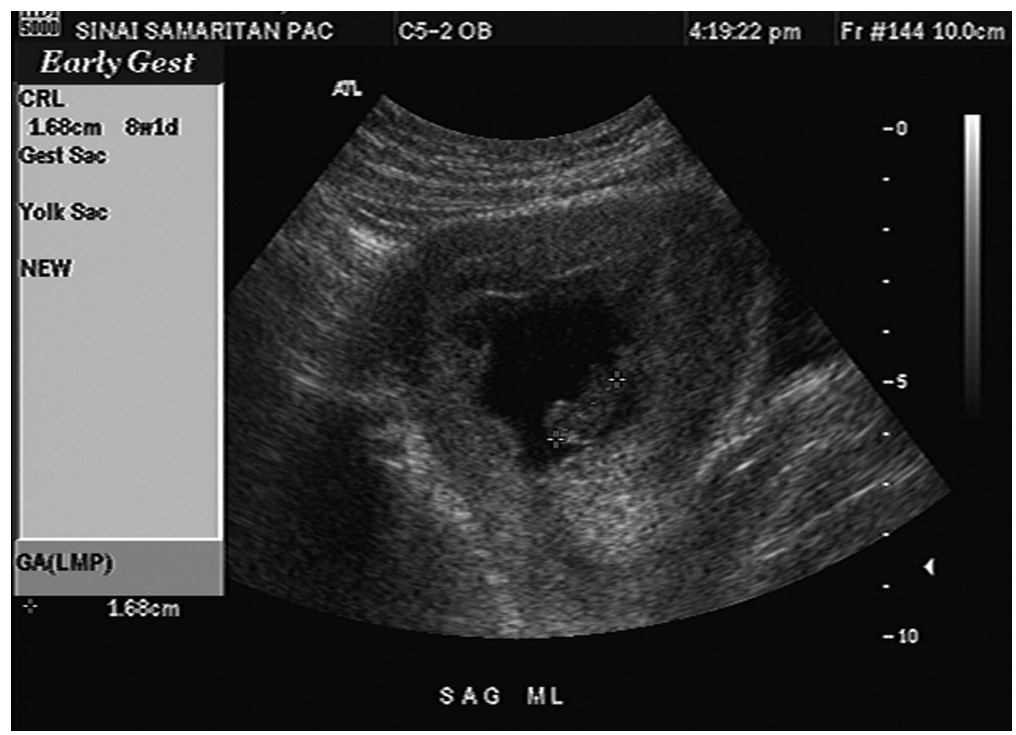

Figure 1. Ultrasound of an irregular intrauterine gestational sac containing an 8-week-sized fetus.

ment, usually because of a delayed or erroneous diagnosis." 2 Heterotopic pregnancies-coexisting intrauterine and ectopic pregnancies-may result in tubal rupture, intra-abdominal bleeding, and death before medical attention can be sought.

To date, there is no standard follow-up for spontaneous, therapeutic, and elective abortions. Many patients probably receive no follow-up, putting them at risk for potentially life-threatening consequences of undetected molar and heterotopic pregnancies. Four parameters can be used in post-abortion monitoring: (1) serum B-hCG levels, (2) sonographic evaluation and measurement of the endometrial thickness or endometrial echo complex (EEC), (3) serial hemoglobin or hematocrit measurements, and (4) bleeding pattern. ${ }^{3}$

Bleeding pattern and hematocrit measurements are not specific and are used principally to decide when to initiate additional testing or treatment. Quantitative B-hCGs and ultrasounds can give earlier and more specific diagnoses for complications after first trimester losses.

The lack of associated signs and symptoms in the patient being discussed is typical for a patient in early pregnancy with a partial mole. Because of earlier diagnoses of molar pregnancies with the widespread use of B-hCGs and ultrasound, theca lutein cysts and hyperthyroidism, consequences of very high B-hCG levels, are rare. ${ }^{4}$

Symptoms of partial moles are less severe than those of complete moles, and diagnosis is often made on histologic examination of uterine con- tents. ${ }^{4}$ Even with complete moles (Figure 2), ${ }^{5}$ hyperemesis, preeclampsia, hyperthyroidism, and ovarian enlargement are rare because of the routine use of transvaginal ultrasound and B-hCGs. ${ }^{6}$

Relevant to the patient being discussed, "several studies have shown that even experienced pathologists may have difficulties in distinguishing this disorder and. . . it has been found that the concordance rate between pathologists for the diagnosis of molar pregnancies (CHM or PHM) ranges from $55 \%$ to $75 \%$." 2 Thus, following B-hCGs after a normal pathology report is indicated for detecting molar pregnancies, in addition to heterotopic pregnancies.

Ultrasonography alone is insufficient for diagnosing molar pregnancies: in a study of 155 molar pregnancies, two thirds were missed on ultrasound. ${ }^{7}$ Judicial use of ultrasound in conjunction with serial B-hCGs may be the best method of preventing the morbidity and mortality of untreated disease after early pregnancy loss.

The ideal frequency of B-hCG testing has not been established. ACOG classifies its recommendation that "abnormal bleeding for more than 6 weeks after any pregnancy should be evaluated with hCG testing to exclude a new pregnancy or gestational trophoblastic disease" as a Level C recommendation-based primarily on consensus and expert opinion. ${ }^{8}$ Better evidence would be helpful in the management of patients who have had a first trimester loss. 


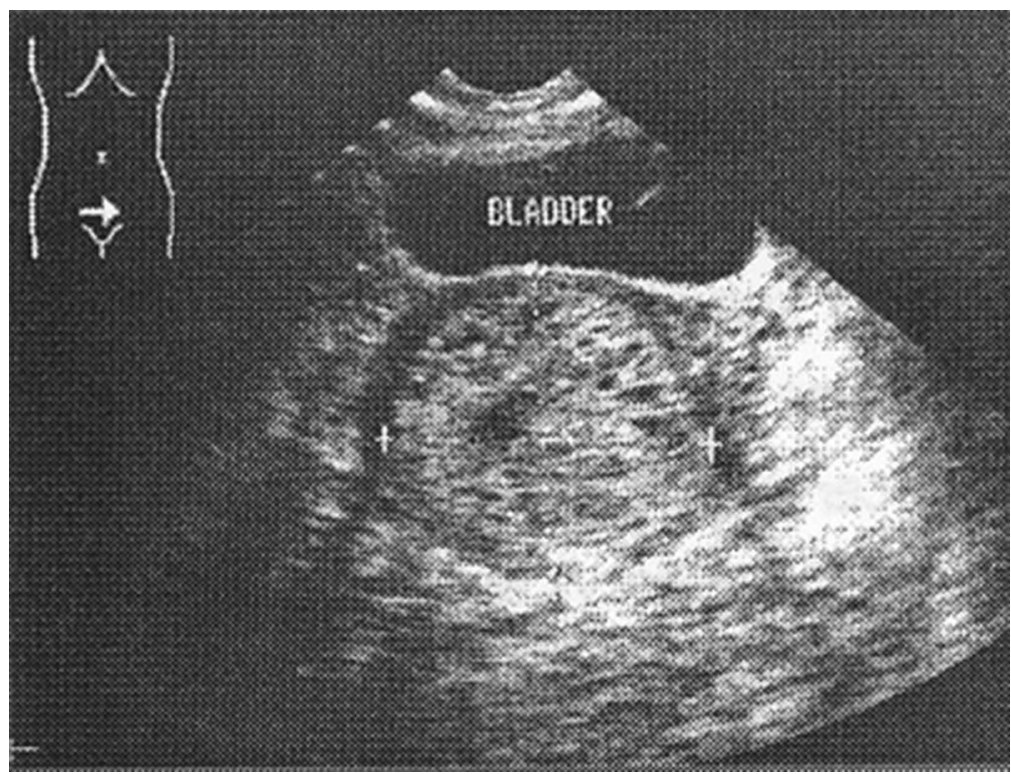

Figure 2. Snowstorm appearance of complete mole on ultrasound.

An argument can be made for more frequent B-hCG testing. Waiting 6 weeks to begin monitoring B-hCGs may be too long for some patients: "generally patients with low risk choriocarcinoma have a good prognosis and mortality is lowest when diagnosed within 6 weeks of a proceeding pregnancy. The mortality increases progressively with increase in time interval between pregnancy and diagnosis. . . A malignancy diagnosed 7 weeks to 3 months after pregnancy carries a mortality rate of $55 \%$ which increases further to $75 \%$ if diagnosis is made 7 months to 5 years after pregnancy." 9 Some providers, including the author of this article, check B-hCGs weekly until a urine pregnancy test is negative after first trimester losses. A cost-benefit analysis would be helpful in recommending the best follow-up regimen. For this, additional research is needed to determine the outcomes of different approaches.

From a cost-benefit perspective, some might argue that it is not worth spending the resources to monitor for relatively infrequent molar and heterotopic pregnancies. The molar pregnancy rate is 1.5 per 1000 live births, and the heterotopic pregnancy rate is 0.03 per 1000 live births. ${ }^{4}$ Heterotopic pregnancy rates are higher, but still relatively low, in patients undergoing assisted reproduction with reported rates as high as 1.1 per $1000 .^{10}$

An argument for close monitoring after first trimester losses can be supported by looking at numbers rather than rates. Lives saved and years of potential life lost are high considering that the United States had 4,021,726 registered births in $2004^{11}$ and the global population is growing by 76 million persons per year. ${ }^{12}$

The risk of recurrence, as occurred with the patient being discussed, increases after having a molar pregnancy. After one mole, the risk is less than 1 in 50 ; after 2 , the risk is 1 in 6 ; and after 3 , the risk is approximately 1 in $2 .^{4}$

\section{Conclusion}

Molar and heterotopic pregnancies may still be present after the confirmed loss of an early intrauterine pregnancy. If missed, both can be lifethreatening. Following serial B-hCG levels after first trimester losses is one effective manner of detecting molar and heterotopic pregnancies. Additional research is needed to determine the optimal follow-up regimen.

\section{References}

1. Mungan T, Kuscu E, Dabakoglu T, Senoz S, Ugur M, Cobanoglu O. Hydatidiform mole: clinical analysis of 310 patients. Int J Gynecol Obstet 1996;52: 233-6.

2. Petignat P, Billieux M, Blouin J, Dahoun S, Vassilakos $\mathrm{P}$. Is genetic analysis useful in the routine management of hydatidiform mole? Hum Reprod 2003; 18:243-9.

3. Harwood B, Meckstroth KR, Mishell DR, Jain JK. Serum beta-human chorionic gonadotropin levels 
and endometrial thickness after medical abortion. Contraception 2001;63:255-6.

4. Hancock BW, Tidy JA. Current management of molar pregnancy. J Reprod Med 2002;47:347-54.

5. Deutchman M, Eisinger S, Kelber M. First trimester pregnancy complications [slide 26], Advanced Life Support in Obstetrics Course Syllabus, 4 ed. Shawnee Mission (KS): American Academy of Family Physicians; 2000.

6. Coukos G, Makrigiannakis A, Chung J, Randall TC, Rubin SC, Benjamin I. Complete hydatidiform mole: a disease with a changing profile. J Reprod Med 1999;44:698-704.

7. Sebire NJ, Makrydimas G, Agnantis NJ, Zagorianakou N, Rees H, Fisher RA. Updated diagnostic criteria for partial and complete hydatidiform moles in early pregnancy. Anticancer Res 2003;23:1723-8.
8. ACOG Practice Bulletin no. 53. Diagnosis and treatment of gestational trophoblastic disease. American College of Obstetricians and Gynecologists: 2004.

9. Saydain G, Raoof S, Khan FA. Solitary large lung mass and amenorrhea in a female smoker. JK-Practitioner 2002;9:244-6.

10. Perkins JD, Mitchell MR. Heterotopic pregnancy in a large inner-city hospital: a report of two cases. J Natl Med Assoc 2004;96:363-6.

11. National Center for Health Statistics. Health, United States, 2004 with chartbook on trends in the health of America. Washington (DC): U.S. Government Printing Office; 2004. Available at http://www. cdc.gov/nchs/data/hus/hus04acc.pdf.

12. United Nations Population Fund. State of world population: 2004. Site last visited March 19, 2005. Available at http://www.unfpa.org/swp/2004/english/ch1/ page7.htm\#1. 